\title{
Estado de salud de las personas sin hogar en Cádiz y construcción de un instrumento de intervención social
}

\section{Gabriel Robles Gavira}

Técnico de Gestión de Programas, Delegación Municipal de Asuntos Sociales del Ayuntamiento de Cádiz y Tutor de Sociología, Centro Asociado de la UNED-Cádiz gabriel.roblesgavira@cadiz.es

\section{Esther Hernández Fernández}

Trabajadora social, Cruz Roja Española-Cádiz

\section{Mónica Cubiella González}

Educadora social, Delegación Municipal de Asuntos Sociales del Ayuntamiento de Cádiz

\begin{abstract}
Artikulu honen helburua Cadizen etxerik gabeko pertsonen osasun-egoera deskribatzea da, gizarteeskuartze tresna bat egiteko. Lehenik eta behin, biztanleria horren osasun-arazoak erakusten dira. Bigarrenik, gizarte- eta osasun-baliabideekin ezartzen duten elkarrekintza. Ikerketa-metodologiak ikuspegi kuantitatiboak eta kualitatiboak konbinatzen ditu. Alde batetik, 2017ko Etxerik Gabeko Pertsonen Erroldako datuak erabiltzen ditu, baita funtsezko informatzaileei eta etxerik gabeko pertsonei egindako elkarrizketa sakonak ere. Azkenik, datuak, diskurtsoak eta kaleko ekintzak aztertzea da eskuartzeko tresna baten etapak egiteko oinarria. Tresna horren osagarriarekin kalean egiten diren eskuartzeek agerian uzten dute kalean bizitzearen arrisku soziosanitarioak murrizteko eta osasunerako duten eskubidea gauzatzen laguntzeko eraginkorrak direla.
\end{abstract}

\section{GAKO-HITZAK:}

Etxerik gabeko pertsonak, arriskuak murriztea, exijentzia txikia, gizarte-eskuartzea, osasuna.
El objetivo del siguiente artículo es describir el estado de salud de las personas sin hogar en Cádiz para elaborar un instrumento de intervención social. En primer lugar, muestra los problemas de salud de esta población. En segundo lugar, la interacción que establecen con los recursos sociales y sanitarios. La metodología de investigación combina los enfoques cuantitativos y cualitativos. Por una parte, utiliza los datos del Censo de Personas sin Hogar de 2017 y las entrevistas en profundidad a informantes clave y personas sin hogar. Por último, el análisis de los datos, los discursos y las acciones en la calle es el fundamento para elaborar las distintas etapas de un instrumento de intervención. Las intervenciones en la calle con el complemento de este instrumento demuestran su eficacia para reducir los riesgos sociosanitarios de vivir en la calle y apoyar a quienes lo hacen en el ejercicio de su derecho a la salud.

\section{Palabras clave:}

Personas sin hogar, reducción de riesgos, baja exigencia, intervención social y salud. 


\section{Introducción}

En las sociedades del bienestar existe un colectivo que está situado en la exclusión social extrema, las personas sin hogar. Son aquellas personas que no pueden conservar un alojamiento adecuado, estable y permanente. Es decir, según la clasificación ETHOS (European Typology on Homelessness and Housing Exclusion), están ubicados en la categoría "Sin techo", viven en la calle o en albergues y pasan la mayor parte del día en espacios públicos (Feantsa, 2017).

Esta situación de vulnerabilidad queda reflejada en su estado de salud. La Estrategia Nacional Integral para Personas sin Hogar 2015-2020 dedica un apartado a la situación sanitaria de las personas sin hogar (MSCBS, 2015:18-21). En ella, menciona tres aspectos relevantes; por una parte, la esperanza de vida de estas personas es de treinta años menor que la población general. En segundo lugar, las muertes de estas personas están asociadas a problemas crónicos de salud más que a la enfermedad mental o al consumo de sustancias. Por último, las personas sin hogar que muestran mayor mortalidad en comparación con el conjunto de la población son los jóvenes y las mujeres, quienes tienen, respectivamente, entre 5,9 y 10 veces más posibilidades de morir. Estos datos manifiestan la fragilidad del estado de salud de este colectivo.

La presente investigación tiene como objetivo general conocer la situación sociosanitaria de las personas sin hogar en la ciudad de Cádiz, entre finales de 2017 y la primera mitad del año 2019. Abarca los siguientes objetivos detallados:

1. Conocer el estado de salud de las personas sin hogar en Cádiz.

2. Analizar la relación entre la situación social de estas personas y la interacción con los recursos sanitarios.

3. Elaborar y presentar un protocolo sintetizado para la intervención sociosanitaria con esta población.

La investigación también propuso una hipótesis respecto al estado de salud de las personas sin hogar. Esta relaciona el deterioro de la salud de estas personas por el tiempo que acumulan en la situación de sinhogarismo, es decir, el tiempo como factor acumulativo en el empeoramiento de sus condiciones físicas y psíquicas, a más tiempo en la calle, mayor deterioro de la salud.

La metodología del estudio combina los enfoques cuantitativos y cualitativos (García, Ibáñez y Alvira, 1993; Alonso, 1998; Corbetta, 2010). En primer lugar, los datos del último censo en Cádiz (Ayuntamiento de Cádiz, 2017) aportan una radiografía cuantitativa de esta población. La información extraída muestra sus características sociodemográficas, así como preguntas específicas sobre su salud y la percepción subjetiva de esta. Además, estos datos relacionan su estado de salud y el tiempo que llevan en la calle. La segunda parte recurre a las entrevistas en profundidad (Alonso, 1998; Valles, 2009) de dos colectivos. De un lado, los profesionales, informantes clave, asociaciones e instituciones que trabajan en este ámbito. Por otro, recopila y analiza la experiencia biográfica de las personas sin hogar.

Por último, la distribución de los datos cuantitativos y el estudio de la significatividad de los discursos constituyen la base para elaborar un protocolo de intervención sociosanitaria con estas personas. El protocolo incluye la descripción de las distintas fases, agentes y tareas concretas para desarrollar la intervención de caso. Además, queda complementado con los requisitos que deben seguir para que obtener la documentación personal que les permita acceder al derecho a la salud (documento nacional de identidad, documento de empadronamiento y tarjeta sanitaria).

\section{Marco teórico y antecedentes}

\subsection{La inspiración de la reducción de riesgos y la baja exigencia}

En esta intervención, el trabajo está unido a los conceptos y principios de la reducción de riesgos y la baja exigencia. Los orígenes de la reducción de riesgos están ligados a la epidemiología, cobrando mayor protagonismo a partir de la segunda mitad del siglo XX (Segura, 2014). Esta teoría considera la influencia de los factores socioculturales y los estilos de vida en los problemas de salud y que derivan en enfermedades graves o crónicas (Segura, 2014). Por otro lado, la reducción de riesgos adquiere popularidad en la década de 1980-90 a través de los programas de reducción del riesgo por consumo de drogas por vía parenteral y a la prevención del contagio del VIH-sida (García y Arévalo, 2015; Calvo et al., 2017). La reducción del daño consiste en aquellos programas, acciones o políticas encaminadas a minimizar las consecuencias negativas asociadas al consumo de drogas (Marlatt, 1996; García y Arévalo, 2015; Calvo et al., 2017).

La reducción de los riesgos implica mejorar en lo posible la vida y la salud de las personas, aunque permanezcan en la calle. El trabajo sociosanitario de reducción de riesgos abarca varias fases y objetivos. Primeramente, respeta los tiempos, los ritmos y los deseos de cada persona. A partir de ahí, propone la creación de un vínculo para lograr el acercamiento al sistema sanitario para conseguir, por ejemplo, la tarjeta sanitaria, tener un profesional de referencia o un centro de salud. En el siguiente paso, el sujeto obtiene el diagnóstico y le asignan un tratamiento. De vuelta a la calle, los profesionales hacen el seguimiento del tratamiento. En lo posible, obtienen las medicinas y las entregan. Por otro lado, supervisan la toma de medicamentos y las siguientes consultas que requiere el proceso terapéutico.

Observan y registran la evolución general de la salud 
del individuo y de las patologías que padece. En el caso de una situación grave, según el caso, gestionan con los servicios sociales las medidas de protección o de apoyo a la dependencia. Transversalmente, deben apoyar el trabajo con un acompañamiento constante y regular, pero siempre con la intención de conseguir progresivamente la autonomía del sujeto.

Paralelos a la reducción de daño están los principios de la baja exigencia. Cuando una persona está física y psicológicamente en un estado muy precario no puede brindar ningún tipo de esfuerzo a cambio de la ayuda (Marina, 2018). Es decir, la baja exigencia establece muy pocos requisitos o contraprestaciones para que las personas accedan a los recursos 0 servicios. En este caso, el programa de intervención sociosanitaria mantiene su apoyo en cualquier circunstancia. Con todo, también respeta la capacidad de decisión y los deseos de las personas. En ese momento, es obligatorio para la sociedad y las instituciones cubrir las necesidades básicas para la vida. Además, una característica de los recursos de baja exigencia es la proximidad a los usuarios (Marina, 2018), la versatilidad, la disponibilidad y el acceso, lo más rápido posible, para responder a circunstancias críticas, que en ocasiones implican la diferencia entre la vida y la muerte.

\subsection{Salud y factores de riesgo en las personas sin hogar}

La Organización Mundial de la Salud en su constitución define la salud como "un estado de completo bienestar físico, mental y social, y no solamente la ausencia de afecciones o enfermedades" (OMS, 1946: 1). La utilidad de esta definición está en que describe la salud como un estado multidimensional, añadiendo la dimensión social a las circunstancias individuales. De ahí que las causas de los problemas de salud haya que abordarlas desde la interdisciplinariedad (Alcántara, 2008). La intervención multidisciplinar es necesaria en el trabajo con personas sin hogar debido a la complejidad de los procesos de exclusión y pobreza extrema. Las causas de la exclusión remiten a factores de riesgo que coadyuvan con la evolución hacia la situación de sinhogarismo, siendo los factores de riesgo aquellas características o atributos contextuales, relacionales o individuales que favorecen la aparición del problema (Becoña, 2002; Moncada, 1997).

El estado de estas personas es el resultado de la sinergia de factores estructurales, relacionales e individuales (Cabrera, 1998; Sánchez, 1999; Subirats, 2004 y 2005; Tezanos, 2008). Los primeros están relacionados con dificultades económicas, laborales, educativas y formativas, institucionales o residenciales. Los factores relacionales remiten a la pérdida de la red de apoyo y los vínculos interpersonales, familiares y comunitarios. Castel (1995) los define como: procesos de desafiliación. Es decir, un progresivo desligamiento y desapego social que le aleja del contacto social. Por último, los factores personales incluyen tanto las características adscritas como las adquiridas. Las adscritas son variables como la edad, el sexo, la personalidad, una discapacidad, etc. Las adquiridas remiten a separaciones, divorcios, adicciones, delincuencia o sucesos vitales estresantes que hacen referencia a experiencias de profunda gravedad emocional que sufre el individuo (Cabrera, Malguesini y López, 2002; Muñoz, Vázquez y Vázquez, 2003; Robles y Pérez, 2014; Matulič, De Vicente y Caïs, 2018).

Estos sucesos vitales traumáticos tienen una especial relevancia entre los sin hogar, sobre todo por la mayor frecuencia y el momento del hecho. La gravedad y la acumulación de estos acontecimientos provocan graves consecuencias físicas y psicológicas que acompañan la trayectoria de los sujetos.

Además, la sucesión de estos incidentes constituye una causa significativa para que las personas entren en situación de sinhogarismo y para que perpetúen la permanencia en la calle (Roca et al., 2019).

\subsection{Desigualdades sociales en salud y personas sin hogar}

En el ámbito de las desigualdades, las diferencias sociales marchan paralelas a las diferencias en salud (Escolar, 2013; Segura, 2014; MSPS, 2010; Llano, 2019). Estas últimas muestran los desniveles en la salud de una población con relación al continuo de las posiciones en la estructura social (Escolar, 2013; Segura, 2014). Las desigualdades en salud analizan la influencia que tienen en los distintos grupos de población variables como la clase social, los ingresos, la ocupación, la vivienda, el urbanismo, la educación, la edad, el sexo, etc. El mal estado de salud está asociado a peores condiciones socioeconómicas; por el contrario, un mejor nivel económico conlleva ventajas, como el acceso rápido a servicios médicos y a los especialistas, la adquisición de medicinas, pautas de prevención o estilos de vida saludables (Escolar, 2013).

En el caso de las personas que viven en la calle, las diferencias sociales y, por tanto, las desigualdades en salud, son relevantes. El hecho de no tener un hogar, un lugar estable y seguro donde pernoctar, los instala en el espacio de la exclusión social severa. Sus propiedades son las pertenencias que pueden transportar. Esta exposición a la intemperie hace que los problemas de salud empeoren y se cronifiquen.

Los problemas de salud entre las personas sin hogar coinciden, sobre todo, con dolencias respiratorias, infecciosas, músculo-esqueléticas y mentales. La investigación de Muñoz, Vázquez y Vázquez (2003) en Madrid destaca las enfermedades del sistema respiratorio (23,5\%), los problemas óseos o musculares (23,5\%), los problemas gastrointestinales (18\%) y los ingresos psiquiátricos (15,7\%). En Barcelona, Uribe y Alonso (2009) describen las dolencias músculo-esqueléticas 
(67,6\%), las cardiovasculares (57\%) y los trastornos mentales ( $49 \%)$. Por otro lado, analizando las causas de los ingresos hospitalarios de las personas sin hogar en los hospitales Virgen Macarena y Virgen del Rocío de Sevilla, los principales problemas que aparecen son los mentales ( $27 \%$ ), en segundo lugar, las enfermedades infecciosas (19,6\%) y en tercero, las respiratorias $(18,4 \%)$ (Torrero, Fernández y Charris, 2016).

Este último estudio, en relación con el consumo de sustancias, señala que el $77,6 \%$ de las personas $\sin$ hogar ingresadas en el hospital consume alguna sustancia, ya sea alcohol, tabaco, opiáceos, cannabis o cocaína (Torrero, Fernández y Charris, 2016). En cambio, la encuesta estatal del Instituto Nacional de Estadística (2012) refleja que, en la población general, el consumo de alcohol es del $44 \%$ (ligero, $30,5 \%$; moderado, $9,5 \%$; alto $1,1 \%$; y excesivo $3 \%$ ) mientras que los abstemios son el $56 \%$. El consumo de drogas tiene una presencia habitual en el colectivo (Panero y Muñoz, 2014) y existe correspondencia con los trastornos mentales (INE, 2012; Feantsa, 2015). Asimismo, está asociado a enfermedades graves derivadas de las prácticas de riesgo por consumo intravenoso, como la hepatitis B o C (40,2\%) y el sida $-30,9 \%-$ (Moreno, 2009). Para terminar, en cuanto a la mortalidad, las causas son la acumulación de problemas de salud, las muertes accidentales y las victimas por violencia o suicidios (Carreras et al., 2017).

\section{Metodología}

\subsection{Investigación-acción y el trabajo con casos}

Esta investigación puede considerarse teórico-práctica o de investigación-acción (Fernández y Ponce de León, 2018). Por una parte, analiza las circunstancias que rodean a la persona, e indaga en las causas y en las trayectorias de exclusión hasta el momento actual. Igualmente, averigua cuáles son las necesidades inmediatas del sujeto para vincularlas a los objetivos de la intervención. Esta retroalimentación entre la experiencia biografía, las demandas personales y los fines de la intervención instaura una sinergia hacia la transformación a largo plazo de la vida del sujeto. Es un proceso de descubrimiento, reconstrucción y progreso. La investigación-acción recurre al marco de una metodología de trabajo social de casos, definido como:

[...] el proceso educativo y social que realiza el profesional con la persona objeto de intervención para que pueda superar las necesidades o problemas sobrevenidos con el objeto de desarrollar habilidades y destrezas que les permitan proseguir de forma autónoma con su proyecto de vida en la dirección marcada por su propia existencia. En otras palabras, lo que debe intentar el profesional es que el usuario modifique o vuelva a trazar un nuevo plan que le facilite conseguir sus objetivos [...]. (Fernández y Ponce de León, 2018: 50)
Todo esto sin olvidar que el análisis e intervención con casos genera regularidades que ayudan a la creación de un protocolo que guíe a los profesionales. Este instrumento quiere acompañar la acción sin limitar la flexibilidad y la adaptación que requieren las singularidades de cada historia. Es decir, que este protocolo es una orientación para la acción no una fórmula inamovible.

\subsection{Fases del proceso metodológico}

La metodología de trabajo combina distintas técnicas. En primer lugar, los datos sociodemográficos del último censo de personas sin hogar en Cádiz del año 2017 (Ayuntamiento de Cádiz, 2017) sirvieron para escoger el perfil de las personas sin hogar a entrevistar. Segundo, la selección y la entrevista a los profesionales que trabajan directamente con personas sin hogar, ya pertenezcan a instituciones de la Administración Pública como al ámbito asociativo. En la siguiente fase, el equipo realiza las entrevistas en profundidad a las personas sin hogar. El trabajo de investigación se extendió desde finales de 2017 hasta los primeros meses del año 2019, desde la puesta en marcha del censo hasta la elaboración final del protocolo de intervención.

\subsection{Entrevistas a informantes clave}

Los informantes clave son aquellas personas que tienen información relevante para los fines de la investigación y son escogidas por su rol preeminente en un determinado ámbito de estudio (Valles, 2009). Este apartado incluye una serie de entrevistas a profesionales e integrantes de organizaciones no gubernamentales que trabajan con personas sin hogar. Estos informantes son escogidos porque disponen de información inmediata, actualizada y una experiencia de largo recorrido con esta población. En muchas ocasiones, estos recursos son los primeros que observan el deterioro de la salud de las personas que frecuentan sus servicios. En esos momentos, realizan una labor de comunicación, acompañamiento o activación, según la gravedad, de los servicios sanitarios de emergencia. Sus discursos son un complemento a los testimonios de las personas sin hogar.

La entrevista persigue conocer la labor del recurso, del profesional entrevistado, su visión de la situación y la evolución sanitaria del colectivo. Una segunda parte dentro del ámbito social se centra en las necesidades que tienen, así como en los puntos fuertes y débiles de su intervención. Concluyendo, la entrevista aborda propuestas de mejora y alternativas de actuación contra el sinhogarismo. El número y selección de los informantes, dependiendo del ámbito en que trabaja y del número de entrevistas, es el siguiente:

- Profesional 1. Recurso de alojamiento.

- Profesional 2. Recurso de alojamiento. 
- Profesional 3. Recurso de manutención.

- Profesional 4. Recurso de manutención.

- Profesional 5. Recurso de intervención en la calle.

- Profesional 6. Recurso de intervención en la calle.

- Profesional 7. Recurso sanitario de ONG.

- Profesional 8. Recurso hospitalario.

- Profesional 9. Recurso de centro de salud.

- Profesional 10. Recurso de salud mental.

\subsection{Población sin hogar y criterios de selección}

La población objeto del estudio comprende las categorías 1 y 2 de la definición ETHOS: "sin techo" y “sin vivienda” (Feantsa, 2017). Los resultados del censo de 2017 recogen 98 personas sin techo/sin vivienda en la ciudad. La distribución por sexos fue del $12,2 \%$ de mujeres y del $82,6 \%$ de hombres. El porcentaje que falta son "no sabe/contesta", porque no se les molesta si están dormidos o no quieren participar. La muestra intencional es de diez sujetos a entrevistar, que corresponde a un $10 \%$ de la población sin hogar, dos mujeres y ocho hombres, algo por encima de la proporción por sexos.

Teniendo en cuenta tanto la heterogeneidad del colectivo estudiado como los distintos perfiles, ha sido necesaria la selección de unos criterios de inclusión y exclusión para poder definir los sujetos del estudio. Los siguientes criterios definen los casos seleccionados:

- Criterios de inclusión:

1. Personas que duermen en la calle de forma sistemática (cajeros, portales, playa, parking, etc.) y aquellas que frecuentan algún recurso asistencial.

2. El tiempo en la calle, porque influye en el progresivo deterioro físico, psíquico y social de estas personas.

3. El sexo, para mantener la proporción de varones y mujeres.

- Criterios de exclusión:

1. Personas con un alto deterioro cognitivo.

2. Personas que rechazan la entrevista.

Una vez seleccionada la población, la elección de los entrevistados queda compuesta por dos mujeres y ocho hombres organizados en función del tiempo sin hogar.

Tabla 1. Selección de los entrevistados por sexo y tiempo en la calle

\begin{tabular}{|l|c|c|}
\hline Tiempo en la calle & Hombres & Mujeres \\
\hline Menos de 2 años & 2 & 1 \\
\hline Entre 2 y 6 años & 2 & 1 \\
\hline Entre 6 y 10 años & 2 & 0 \\
\hline Más de 10 años & 2 & 0 \\
\hline
\end{tabular}

Fuente: Elaboración propia.

\subsection{Entrevista a las personas sin hogar}

La entrevista es semiestructurada pero dinámica y flexible, con una duración de entre una hora y hora y media. Son programadas con antelación para no invadir la intimidad de estas personas y para que puedan responder de manera voluntaria y abierta a las preguntas realizadas. Por este motivo, algunas preguntas son modificadas o adaptadas según el desarrollo de la propia entrevista ya que cada participante tiene un margen de tolerancia distinto 0 tiende a responder de manera personal.

Estas son las características de las personas sin hogar entrevistadas -las entrevistas están precedidas de las siglas PSH (personas sin hogar).

1. Hombre, 66 años, menos de 2 años en la calle.

2. Mujer, 49 años, menos de 2 años en la calle.

3. Hombre, 46 años, menos de 2 años en la calle.

4. Mujer, 55 años, de 2 a 6 años en la calle.

5. Hombre, 48 años, de 2 a 6 años en la calle.

6. Hombre, 60 años, de 2 a 6 años en la calle.

7. Hombre, 48 años, de 6 a 10 años en la calle.

8. Hombre, 60 años, de 6 a 10 años en la calle.

9. Hombre, 67 años, más de 10 años en la calle.

10. Hombre, 38 años, más de 10 años en la calle.

\subsection{Intervenciones de calle}

El proceso de investigación-acción ha realizado más de 80 intervenciones de calle. Estas salidas aportan información sobre la realidad cotidiana de este fenómeno, además de constituir una importante experiencia para incorporarla al estudio y a las intervenciones. Son avisos sobre casos de personas sin hogar que llegan a través de los canales entrada que recoge el protocolo (Figura 2).

A continuación, se ofrece la descripción cuantitativa de las intervenciones.

- Caso 1: mujer, 35 años, 3 intervenciones.

- Caso 2: varón, 60 años, 31 intervenciones.

- Caso 3: mujer, 58 años, 8 intervenciones.

- Caso 4: varón, 38 años, 20 intervenciones.

- Caso 5: varón, entre 50-55 años, 2 intervenciones.

- Caso 6: varón, 30 años, 1 intervención.

- Caso 7: varón, 59 años, 1 intervención.

- Caso 8: varón, entre 40-45 años, 2 intervenciones.

- Caso 9: varón, 40 años, 2 intervenciones.

- Caso 10: varón, 53 años, 3 intervenciones.

- Caso 11: varón, 35 años, 1 intervención.

- Caso 12: varón, 40 años, 5 intervenciones.

- Intervención comunitaria 13: Zona C/Doctores Meléndez, 2 intervenciones, asistencia a una reunión monográfica con vecinos y otras delegaciones municipales para programar una intervención con el objetivo de reducir los conflictos generados por la concentración de personas sin hogar en una calle.

- Intervención comunitaria 14: 5 rutas de calle. 


\section{Resultados}

\subsection{La salud de las personas sin hogar}

Cada dos años, desde 2010, las entidades que trabajan en este campo realizan un censo coordinado y sistemático de las personas en situación de sin hogar en la ciudad de Cádiz. El recuento utiliza la categoría 1 de ETHOS, aquellas personas que viven en un espacio público o albergue y/o están forzados a pasar toda la jornada en un espacio público (Feantsa, 2017). El sinhogarismo constituye una realidad compleja y oculta que obliga a los profesionales a revisar los datos con una cierta periodicidad para tener una imagen lo más actualizada posible.

Los últimos datos disponibles de la situación sanitaria de las personas sin hogar en Cádiz quedan recogidos en el Censo de Personas sin Hogar de 2017. En la edición de 2017, el censo contabilizó 98 personas sin hogar. En 2015 fueron 115 personas, en 2013 se hallaron 107 personas y en 2010 fueron 83 personas.

La entrevista pregunta por el número de meses que llevan en la calle. Los estadísticos de tendencia central presentan indicadores altos de tiempo sin hogar. La media alcanza los 7 años y dos meses, la mediana supera los 3 años y las modas, hay dos, se sitúan en los 2 y los 3 años. Es una población con mucho tiempo en el sinhogarismo y envejecida. La edad media de edad está por encima de los 48 años y la mediana en los 50 años.

Agrupando en tramos el tiempo transcurrido en la calle, la población queda distribuida de la siguiente forma: de los 55 que respondieron, cerca de la mitad de los encuestados están ubicados en los dos extremos, menos de un año y más de diez, son recién llegados o están cronificados.

El estado de salud subjetivo es el testimonio personal sobre la autopercepción que tiene un individuo sobre cómo se encuentran al margen de los datos objetivos de un diagnóstico médico. En él influye las enfermedades biográficas y el estado físico del sujeto pero también el entorno socioeconómico y residencial (Pérez y Abellán, 2018). Preguntando por el estado de su salud percibida, consideran que es de regular hacia mejor. Uno de cada tres declara que su estado de salud es "Bueno" y cerca de un 11,2\% dice que es "Muy Bueno". En el lado contrario, entre “Malo"y "Muy Malo", suman cerca del 13,2\%.

Al cruzar el estado de salud con el tiempo que llevan en la calle, puede apreciarse cómo influye la variable. Lógicamente, los recién llegados se sienten mejor, mientras que a medida que avanzamos desciende la percepción positiva de la salud y crecen las opciones más pesimistas, "Regular”, “Malo” y “Muy Malo”, a partir de los tres años.

A las personas que contestaron que su estado de salud era "Regular", "Malo" o "Muy Malo", se les solicitó que identificaran la causa. De las 13 razones que mencionaron, un tercio tenía relación con la salud mental. Los síntomas que alegaron para describir su malestar son: depresión, ansiedad o, genéricamente, problemas mentales. Luego había una larga lista de dolencias muy diferentes que comprenden las siguientes: enfermedades digestivas; enfermedades mentales y del sistema nervioso; enfermedades óseas y musculares; enfermedades del sistema respiratorio; y alcoholismo y otras drogas.

Tabla 2. Tiempo como personas sin hogar

\begin{tabular}{|c|c|c|c|c|c|}
\hline Censo & Perdidos & Válidos & Media & Modas & Mediana \\
\hline 2017 & 42 Casos & 56 Casos & 7,2 años & 2 y 3 años & 3.3 años \\
\hline
\end{tabular}

Fuente: Elaboración propia a partir del Censo de Personas sin Hogar 2017, Ayuntamiento de Cádiz.

Gráfico 1. Porcentaje sobre el estado de salud subjetivo por tiempo en la calle de las personas sin hogar

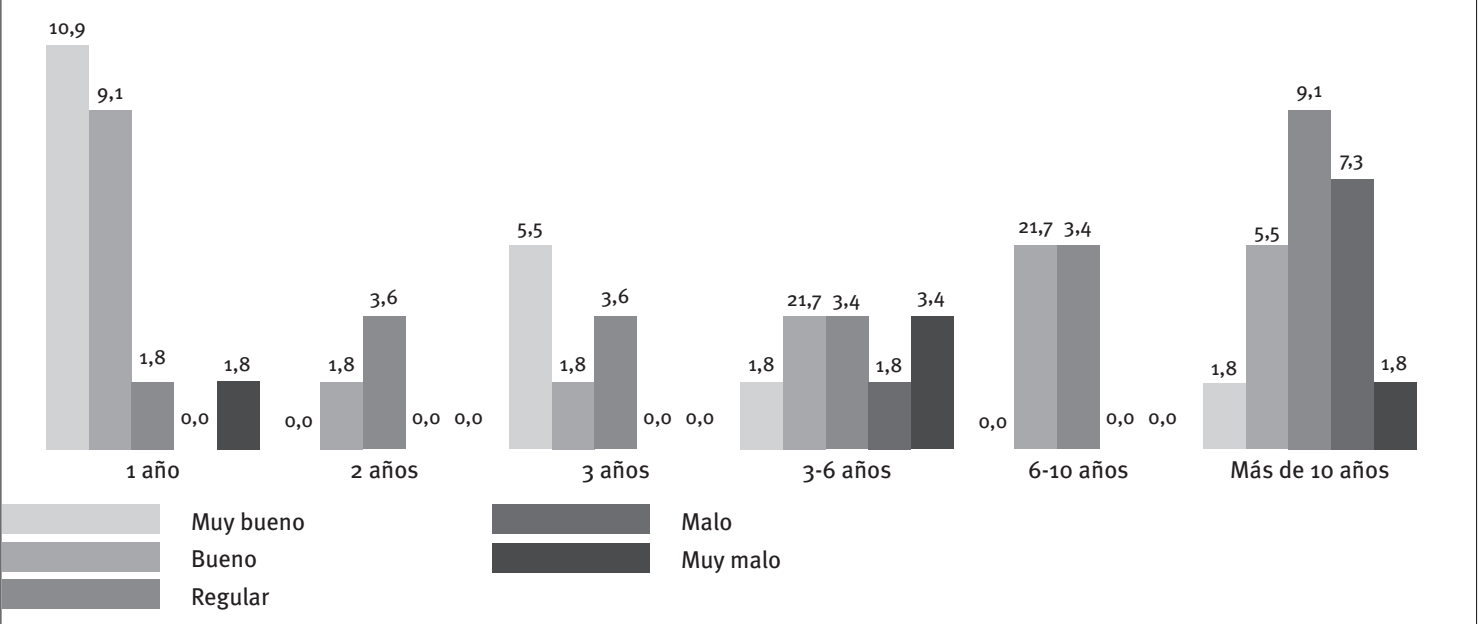

Fuente: Elaboración propia a partir del Censo de Personas sin Hogar 2017, Ayuntamiento de Cádiz. 
Muchas de estas patologías están asociadas al estilo de vida y al tiempo que lleva una persona en la calle. En un principio, los problemas de salud relacionados con esta forma de vida comienzan siendo menores, pero, a largo plazo, si no reciben tratamiento derivan en patologías graves. Por ejemplo, la mala alimentación, el mal estado bucal o el consumo de alcohol propician la aparición de patologías gástricas como gastritis, úlceras, cirrosis, pancreatitis, etc.

Ha habido casos de personas que llevaban seis meses enfermas, decían que no tenían nada y al final tenían un tumor. Un hombre con un cáncer de próstata muy avanzado, que si se hubiese cogido a tiempo se hubiese salvado y, al final, la persona murió. (Profesional 11. Recurso intervención de calle)

En cuanto a al tratamiento y la medicación que reciben, de las 98 personas encuestadas hay que resaltar que un $45 \%$ no responde. Mientras, un $33,7 \%$ manifiesta que no recibe medicación. Solo un pequeño porcentaje, una de cada cinco personas sin hogar, dispone de medicación.

En este sentido, hay que tener en cuenta que la prescripción y adquisición de la medicación por parte de estas personas es difícil y costosa. Por otra parte, en ocasiones el paciente hace un uso indebido, bien por abuso de su consumo, bien por olvido de las tomas o por un abandono total del tratamiento. Asimismo, en conversaciones informales, hacen comentarios sobre "menudeo" con los fármacos para obtener beneficio.

A veces hay un control de la medicación, aunque ellos ya son mayorcitos y son un desastre. Ellos, los mayorcitos, porque a veces no se medican o lo hacen a destiempo. (Profesional 12. Recurso intervención de calle)

Por otro lado, la relación de estas dos variables, tratamiento médico por tiempo sin hogar, es más irregular. Entre los sujetos que siguen el tratamiento decrece, para luego aumentar con los años. Los que responden "No" muestran una curva inversa que remonta cuando pasan de los diez años. Es posible que empiecen a sentir limitaciones físicas y hacen un esfuerzo por recuperar las fuerzas.

Según las respuestas, visitan al médico más o menos la mitad. La otra mitad no responde. De las 44 personas que contestaron, un tercio dijo que dentro de los últimos seis meses. Cerca del $35 \%$ fue a consulta en el último año. La media de meses en acudir se sitúa cerca de los seis meses. La falta de control médico es otra causa que puede favorecer el deterioro de la salud por vivir en la calle.

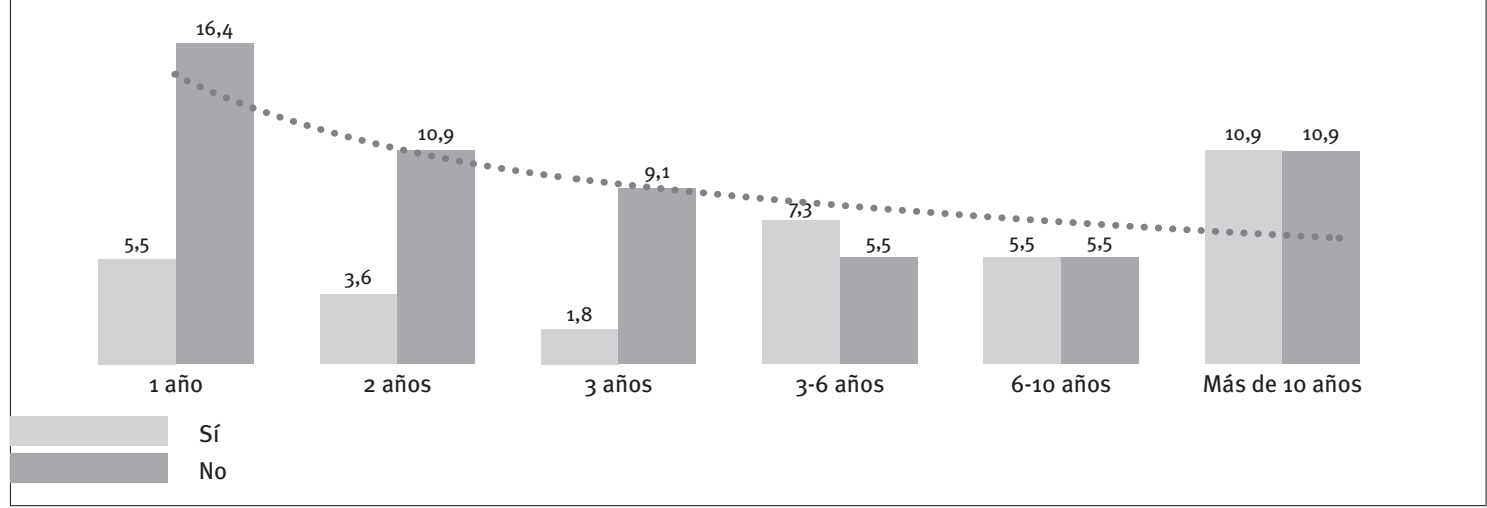

Fuente: Elaboración propia a partir del Censo de Personas sin Hogar 2017, Ayuntamiento de Cádiz.

\section{Gráfico 3. Porcentaje de la última consulta médica por tiempo en la calle}

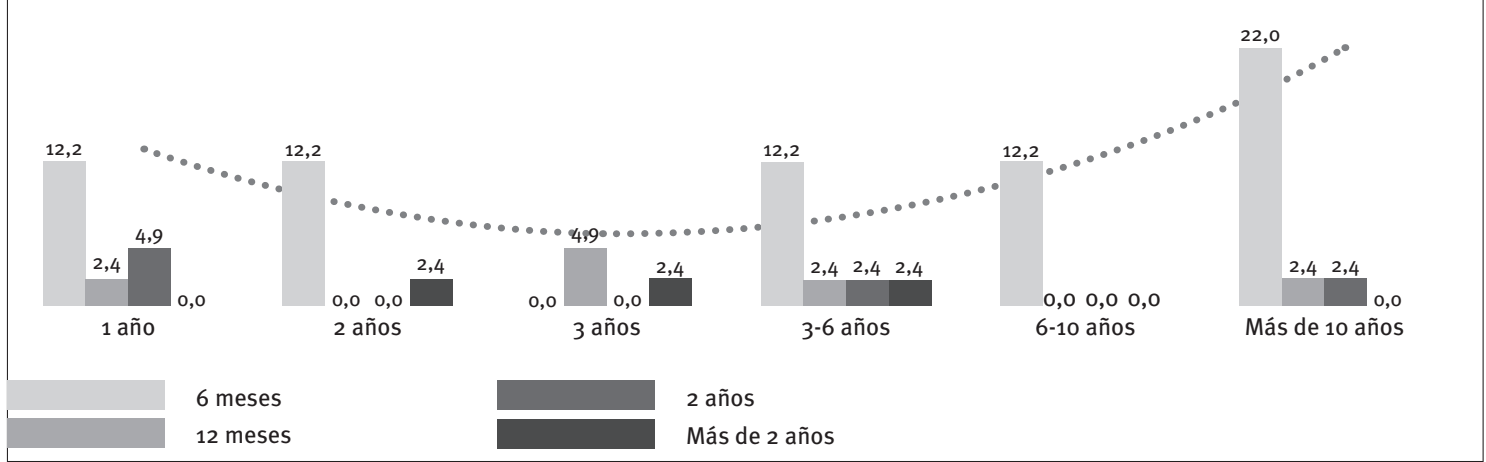

Fuente: Elaboración propia a partir del Censo de Personas sin Hogar 2017, Ayuntamiento de Cádiz. 


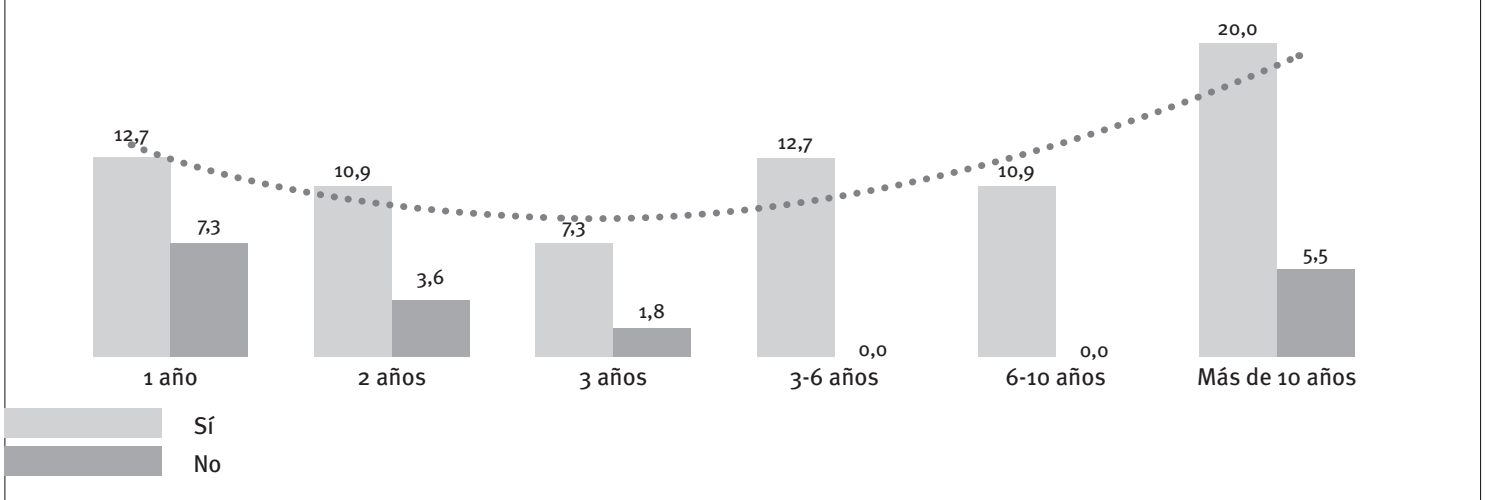

Fuente: Elaboración propia a partir del Censo de Personas sin Hogar 2017, Ayuntamiento de Cádiz.

Con respecto a la tarjeta sanitaria, el documento identificativo de usuario del Sistema Andaluz de Salud, la mayoría de los encuestados la poseen y un $11,2 \%$ no la tienen. Hay un porcentaje alto de no respuesta, cerca del $44 \%$. Hay que averiguar por qué no la tienen y facilitar la documentación a todos y todas, porque la cobertura sanitaria es un derecho social básico.

\subsection{La situación social de las personas sin hogar y el acceso a los recursos}

La dinámica de las personas sin hogar con relación a su salud es que prestan mayor atención a prioridades como la alimentación y el alojamiento. Estas son necesidades básicas diarias. La experiencia con varios casos es que la salud adquiere importancia en el momento en que resulta físicamente limitante. Los profesionales entrevistados comentan al respecto que:

Cuando una persona se encuentra en situación de sin hogar, sus prioridades fundamentales son la búsqueda de un lugar seguro donde dormir y cómo alimentarse cada día, la salud es algo secundario, deja de tener importancia. (Profesional 9. Recurso Centro de Salud)

Por otro lado, las barreras y obstáculos que tienen que sortear para acudir a una consulta provocan que desistan. Los problemas en la adhesión y seguimiento del tratamiento están asociados a su condición de sin hogar. La vida itinerante apunta a que recurren a los servicios médicos de forma improvisada y a través de las urgencias, lo que dificulta la continuidad de la intervención.

El seguimiento estando en la calle es muy difícil; tienes que acudir a urgencias 0 al ambulatorio por desplazados si te encuentras mal, pero un seguimiento por un médico de cabecera sin tener domicilio fijo es muy complicado. (Persona sin hogar 2, hombre, 48 años, entre 2 y 6 años en la calle)

Por sus carencias de notificación, pierden las citas y no recuerdan las fechas programadas, por lo cual casi siempre se salen del sistema sanitario. (Profesional 8, recurso hospitalario)

En relación con lo anterior, los efectos secundarios de un tratamiento errático o la automedicación conllevan una multiplicación de los riesgos que pueden agravar la enfermedad; especialmente problemático es el trastorno mental grave. Si a esto unimos el consumo de alcohol u otras sustancias, los efectos en su salud son manifiestos a nivel físico y psíquico.

Estuve tomando 25 miligramos de tranxilium y fui reduciéndolo hasta que me quedó el tranxilium pediátrico, que lo sigo tomando, que ahora no puedo dejarlo porque si no, no puedo dormir. (Persona sin hogar 2, hombre, 48 años, de 2 a 6 años en la calle)

La calle es muy mala, en el sentido de que la gente como no tiene nada que hacer, está siempre pensando en lo negativo, no en lo positivo y a consecuencia de esto hay tantos robos en la calle, la gente toma tanta droga y la gente está en el alcohol porque de algún modo tienen que pasar el día. (Persona sin hogar 8, hombre, 60 años, entre 6 y 10 años en la calle)

En el anterior comentario, una persona sin hogar muestra la funcionalidad del consumo de drogas. Las sustancias ayudan a conciliar el sueño y también a alejarse de la realidad de la calle. La utilización de ambas opciones combina los fines terapéuticos, evasivos y recreativos, todo en uno. El alcohol libera, los tranquilizantes relajan y, como cuenta un entrevistado, el cannabis es el sustituto ilegal para dormir: "Yo por las noches tengo que tener un par de porros en el bolsillo porque si no, no duermo, me pongo muy mal con los nervios" (Persona sin hogar 10, hombre, 38 años, más de 10 años en la calle). Un efecto asociado al consumo de drogas y medicamentos es el "trapicheo", según relatan, como forma de ganarse la vida.

A nivel afectivo-sexual la tendencia generalizada es la discreción. Son muy reacios a expresarse 
sobre este tema por razonas de intimidad y pudor. Reconocen que tienen información sobre los métodos anticonceptivos. Sin embargo, las respuestas, huidizas y con monosílabos, reflejan la ausencia de consultas y/o controles sanitarios, ya sean ginecológicos, urológicos o programas de información y prevención de enfermedades de transmisión sexual. Por otro lado, la mayoría de las personas sin hogar no tienen pareja. Los hombres confiesan que mantener una relación en la calle implica dificultades porque supone más riesgo y una responsabilidad para con la pareja.

En cuanto a los hábitos de salud cotidianos, los discursos muestran las dificultades para cubrir adecuadamente las necesidades de alimentación, higiene y sueño. La alimentación depende de los comedores sociales o de la solidaridad de la ciudadanía con las personas concretas con las que han establecido un vínculo. En caso contrario, la dieta está basada en bocadillos. La higiene la cubren los dos albergues para las personas alojadas. Además, el Centro de Acogida Municipal tiene un turno de duchas de mañana para los no alojados. En este recurso pueden ducharse y reciben el material para su aseo.

Utilizo las duchas del albergue de seis de la tarde a diez de la noche cuando voy a dormir, están muy bien te lo dan todo allí, maquinilla de afeitar, espuma, las toallas, todo. (Persona sin hogar 4. hombre, 46 años, menos de 2 años en la calle)

En el albergue no hay problemas, a cualquier hora te puedes duchar desde las seis hasta las once, incluso por la mañana para gente de la calle las pueden utilizar. (Persona sin hogar 3, hombre, 66 años, menos de 2 años en la calle)

Sin embargo, otras personas no recurren a los albergues por diferentes motivos. En esos casos, las limitaciones son más importantes y deben recurrir a las estaciones, al hospital, cafeterías, etc. El siguiente comentario es descriptivo al respecto: "Cuando no uso los albergues, tengo que ir a los lavabos de una estación y yo he cogido resfriados gordos por lavarme con agua fría y peinarme con agua fría; temo a los inviernos" (Persona sin hogar 2, hombre, 48 años, entre 2 y 6 años en la calle).

Hay personas sin hogar que no hacen uso de los recursos existentes para pernoctar, aunque se observa un incremento de la utilización de estos espacios durante la temporada de invierno. El hecho de dormir en la calle afecta al estado de salud de las personas. El descanso y el sueño son un factor más. El sueño es intermitente y no mantiene un patrón estable, con lo cual no es un sueño reparador. Por otro lado, el miedo a ser sorprendidos y que puedan sufrir una agresión significa que el estado de alerta está siempre activado. Asimismo, como se ha señalado anteriormente, las personas sin hogar recurren al consumo de alcohol y sustancias tóxicas para conciliar el sueño, lo que a la larga produce otros problemas.
Cuando duermes en la calle, tienes que dormir con un ojo abierto y otro cerrado, y si es con los dos abiertos pues mejor, porque no sabes quién puede llegar, y la policía siempre son tardíos para llegar, y eso afecta a tu estado de salud mental y físico. (Persona sin hogar 2, hombre, 48 años, entre 2 y 6 años en la calle)

Por otro lado, existen dificultades para la intervención y tratamiento de las personas con enfermedad mental grave. Ante estas situaciones, hay un gran desconocimiento sobre cómo afrontarlas. El profesional de lo social no dispone de recursos de emergencia o apoyo en la calle que faciliten la intervención o la derivación, o que simplifiquen los procedimientos cuando la persona se niega rotundamente a colaborar. En ocasiones, los estados de enajenación son tan extremos que constituyen un riesgo para su propia salud y para los demás. Por el contrario, la opción judicial para incapacitar temporal o permanente es un recorrido a medio o largo plazo y como último recurso.

Otro aspecto es la coordinación entre los recursos, que resulta complicada. Los servicios corresponden a diferentes Administraciones; por esto, las prioridades son diferentes y la disponibilidad es limitada.

Hay falta de coordinación entre los servicios sociales; todos aportan cosas, pero de manera libre, no coordinada. (Profesional 1. Recurso intervención de calle)

Con todo, aunque los obstáculos están presentes, el desafío de los profesionales es enfrentarse a ellos. El reto es construir una red de recursos disponible, flexible y eficaz ya que la experiencia, los conocimientos y la voluntad están presentes. Es posible que la respuesta se encuentre en crear una unidad interdisciplinar que afronte la problemática de los casos in situ.

Por último, está el tema de la documentación personal. Un problema recurrente para una parte de las personas que viven en la calle es la falta de documentación (DNI, empadronamiento y tarjeta sanitaria). La ausencia de estos documentos dificulta el acceso sanitario, el diagnóstico precoz y, por tanto, el tratamiento, agravando su situación. El empadronamiento es fundamental para optar a la atención sanitaria en un centro de salud y la asignación de médico. En estos casos, el Centro de Acogida Municipal los empadrona para iniciar el acceso a sus derechos. Los requisitos son demostrar más de seis meses en la ciudad y entrevistarse con la trabajadora social de dicho centro. Las personas que no tienen empadronamiento ni tarjeta sanitaria reciben atención por un año (tarjeta de reconocimiento temporal del derecho a la asistencia sanitaria) con opción de renovación por el médico de referencia. El proceso sintetizado de obtención de la documentación personal queda recogido en la Figura 1. 


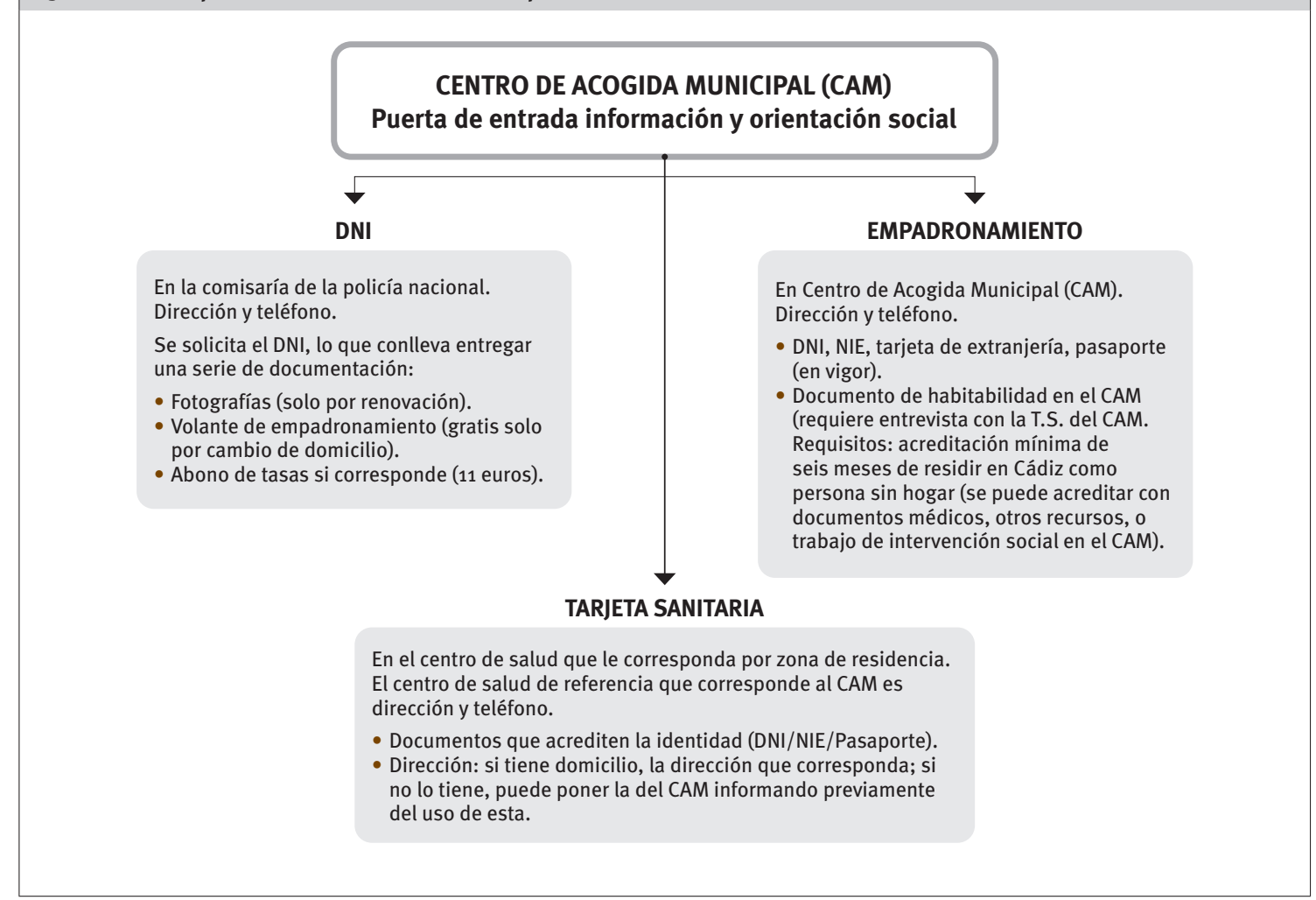

Fuente: Elaboración propia.

\subsection{El protocolo de intervención sociosanitaria con personas sin hogar}

El interés para realizar una investigación sobre las condiciones de salud de la población sin hogar de Cádiz arranca de las reuniones de preparación del Plan de Inclusión Social para Personas sin Hogar por parte de la Mesa de Trabajo sobre Personas sin Hogar. La mesa incluye a entidades sociales de este ámbito, instituciones públicas cuyo trabajo tiene relación con las personas sin hogar (salud mental, drogodependencias, centros de salud, hospital, etc.) y los partidos políticos con representación municipal. Entre las prioridades que incluye el plan hay un apartado que tiene como objetivo recabar información actualizada del fenómeno del sinhogarismo y su evolución en la ciudad.

A comienzos de 2015, el Centro Universitario de Enfermería Salus Infirmorum, adscrito a la Universidad de Cádiz, hace una propuesta de colaboración a la mesa para sondear la situación sanitaria de esta población. A partir de esta petición comienza un proceso que culmina con la inclusión de un apartado específico sobre salud en el censo en sesión de 10 de abril de 2018. Posteriormente, es ratificado en la Junta de Gobierno Local del Ayuntamiento de Cádiz celebrada el día 11 de mayo de 2018. El Plan está organizado en tres dimensiones o programas (relacional y comunitario; intervención socioeconómica y atención individual y sectorial) con sus respectivos proyectos y acciones. La dimensión relacional y comunitaria engloba el proyecto sobre diagnóstico e investigación. En cuanto a las acciones, primero recoge la realización bianual del Censo de Personas sin Hogar. En segundo lugar, propone investigaciones sobre aspectos específicos que profundicen en el conocimiento científico del sinhogarismo. En este apartado es donde encaja el estudio de la situación sociosanitaria de las personas sin hogar.

El plan también acuerda como objetivo implementar protocolos conjuntos con otras instituciones para agilizar la detección e intervención en casos de enfermedad física u orgánica así como con situaciones de enfermedad mental grave. El primero en coordinación con los trabajadores sociales de los centros de salud, y el segundo con la unidad de salud mental de la ciudad. La herramienta de intervención sociosanitaria resultado de esta investigación constituye la estructura sobre la que trabajar con ambas instituciones, incorporando las particularidades de sus distintos campos de estudio.

Este protocolo es el resultado de la formalización de la experiencia adquirida y del análisis de 
las entrevistas. Es una síntesis para guiar las intervenciones. La intervención comienza con la llegada de un aviso a través de diferentes cauces y agentes, tanto institucionales como por ciudadanos particulares. El aviso, según los datos disponibles, es recogido en un boletín informativo que sirve para programar la primera intervención. A continuación, se procede a la búsqueda y localización.

La primera aproximación es de toma de contacto, presentación y exploración de los datos más básicos. El esfuerzo en esta fase consiste en ganar la confianza de las personas y construir un vínculo. Los sucesivos contactos recaban toda la información posible sobre los aspectos biográficos, sociales y sanitarios. Además, se establece contacto con otros recursos sociales para, en caso de que dispongan de información, completar el expediente. Con toda la información comienza a establecerse un plan de acción con las necesidades y objetivos de la intervención. A continuación, el expediente es presentado a la Mesa de Trabajo de Casos, compuesta por las entidades e instituciones que trabajan en el ámbito de las personas sin hogar en la ciudad, para la coordinación, participación o derivación a un recurso más adecuado.

Las siguientes fases son de acompañamiento y seguimiento del proceso. La revisión y adaptación de los objetivos y necesidades es constante para afinar el trabajo. En el caso de alcanzar los objetivos, la intervención pasa a fase de seguimiento, que comprenderá presentación de informes en la Mesa de Trabajo de Casos en la siguiente reunión propuesta, a los tres meses y a los nueve meses. Para terminar, la evaluación final y el correspondiente informe dan por concluido el caso. De no conseguirse los objetivos, debe continuar el seguimiento, modificarse los objetivos y reestructurarse la intervención. La Figura 2 condensa las diferentes fases.

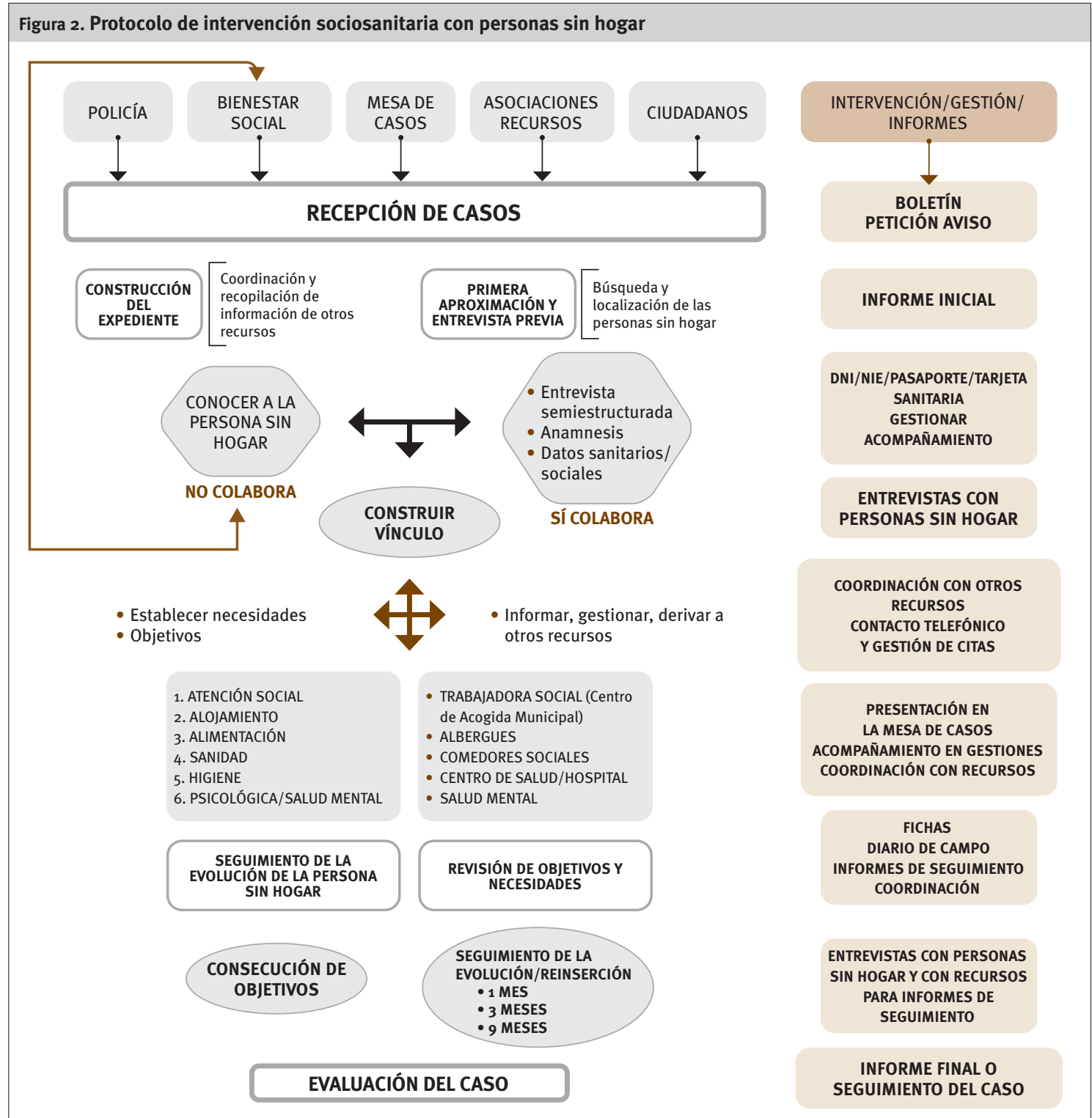

Fuente: Elaboración propia. 
Las dificultades que enfrentan las intervenciones en salud tienen dos vertientes. Primero, por el momento, debido a las contingencias provocadas por la pandemia, la formalización de los protocolos con los servicios sanitarios queda suspendidos. La sobrecarga que padecen los recursos fuerza su retraso hasta que reduzca la presión asistencial.

Por otra parte, después del confinamiento el equipo de calle comenzó a implementar paulatinamente el protocolo de intervenciones sociosanitarias. Entre julio y octubre la recepción mensual superó la media de los cien boletines informativos. Entre las distintas gestiones (de documentación, de ayudas económicas, de vivienda, laborales, demandas ciudadanas, etc.), los temas sanitarios alcanzan las cincuenta intervenciones para el total del periodo. Estas consisten en la solicitud de citas y acompañamiento al centro de salud, control del tratamiento, llamadas a emergencia ante situaciones de riesgo y visitas de seguimiento en el caso de ingresos hospitalarios.

Los datos recogidos hasta el momento y las evaluaciones de los profesionales no cuestionan la metodología, pero sí la extensión y la intensidad del trabajo de calle que es necesario llevar a cabo en estas intervenciones. La construcción y fortalecimiento del vínculo de confianza requiere muchas interacciones. Es una labor extensiva. En cuanto a su intensidad, los profesionales hacen una transición con estas personas desde un estado de aislamiento social hacia la adquisición de confianza y apertura con el profesional. Por otro lado, los tratamientos médicos son largos. Todo esto significa tiempo, esfuerzo y más recursos. Sin embargo, los procesos a largo plazo y los escasos recursos son obstáculos fundamentales para abordar el sinhogarismo.

\section{Conclusiones}

Los datos, los testimonios y las experiencias acumuladas durante esta investigación confirman que la vida en la calle es difícil. Además, cuanto más tiempo permanezca una persona soportando esas condiciones, mayor es el perjuicio para su salud. El paso del tiempo provoca un desgaste físico y psíquico evidente. Su salud percibida es peor con la acumulación de años en la calle. Aparecen dolencias que pasan a ser crónicas. Hay un peor diagnóstico de las enfermedades que puede derivar en problemas graves. Las consultas médicas, las medicinas y/o mantener el tratamiento resulta complicado. La mala alimentación, los problemas de sueño, el descanso poco reparador o la falta de higiene minan las fuerzas y las defensas del individuo, aumentando los riesgos para su salud.

La vida en la calle es un factor de riesgo que afecta a diversas esferas de la vida de los sujetos. Esto se concreta, a nivel físico, problemas digestivos, óseos y musculares, respiratorios y de adicciones a sustancias. Relacionado con lo anterior, el impacto físico y el progresivo aislamiento al que están sometidos repercute en su salud mental y tiene impacto directo en sus capacidades cognitivas (Fundación Arrels, 2017).

La intervención en la calle demuestra ser un factor de protección valioso porque, en primer lugar, consigue información actualizada del estado de salud del sujeto; en segundo lugar, realiza una primera intervención in situ y obtiene datos inmediatos; y tercero, porque inicia el proceso de acompañamiento, derivación o coordinación con otros recursos en el momento de la intervención. Por último, con las intervenciones a medio o largo plazo el seguimiento del caso es sistemático, habitualmente de forma semanal. Gracias al apoyo del trabajo de calle reciben ayuda para acceder a la tarjeta sanitaria y a las consultas médicas. Las intervenciones de calle son útiles para conseguir su adhesión al tratamiento médico. Por último, mejoran la evolución de las enfermedades y su aspecto físico.

Por otro lado, la baja exigencia es un principio que guía toda la intervención. Sin embargo, la experiencia demuestra que no puede aplicarse por igual a todos los casos. Si bien la exigencia tiene que ser más estricta y constante en los casos de sinhogarismo más recientes, por el contrario, debe ser más relajada con los casos que presentaban una mayor precariedad ya que, para ellos, es difícil cumplir con los requerimientos o contraprestaciones de los diferentes recursos. La negociación y la flexibilidad son fundamentales para evitar el distanciamiento del vínculo creado.

Con todo, los resultados de la investigaciónacción han sido positivos, mostrando avances en la recuperación de casos crónicos así como en la incorporación a los recursos y a los procesos de trabajo para la reinserción social de otros casos menos cronificados. 


\section{Bibliografía referenciada}

ALCÁNTARA MORENO, G. (2008): “La definición de salud de la Organización Mundial de la Salud y la interdisciplinariedad", Sapiens. Revista Universitaria de Investigación, 9 (1), pp. 93-107.

ALONSO, L.E. (1998): La mirada cualitativa en sociología, Madrid, Fundamentos.

AYUNTAMIENTO DE CÁDIZ (2017): Informe del censo de personas sin hogar en la ciudad de Cádiz de 2017, Cádiz, Delegación Municipal de Asuntos Sociales (no publicado).

- (2018): Plan de Inclusión Social para Personas sin Hogar, Cádiz, Delegación Municipal de Asuntos Sociales, [no publicado].

BECOÑA, E. (2002): Bases científicas de la prevención de las drogodependencias, Madrid, Delegación del Gobierno para el Plan Nacional sobre Drogas [disponible en: 〈http://www.pnsd.mscbs.gob. es/profesionales/publicaciones/catalogo/ catalogoPNSD/publicaciones/pdf/Bases_ cientificas.pdf $\rangle]$.

CABRERA, P. (1998): Huéspedes del aire. Sociología de las personas sin hogar de Madrid, Madrid, Universidad Pontificia de Comillas.

CABRERA, P; MALGUESINI, G. y LÓPEZ, J.A. (2002): Un techo y un futuro. Buenas prácticas de intervención social con personas sin hogar, Barcelona, Icaria.

CALVO, F.; CARBONELL, X.; GIRALT, C.; LLOBERAS, À. y TURRO, O. (2017): "Reducción de daños asociados al consumo inyectado de drogas en población sin-hogar: Propuesta para una intervención grupal a través de WhatsApp", Pedagogia i Treball Social. Revista de ciències socials aplicades, Vol. 6, Núm. 2, pp. 3-31.

CARRERAS, B.; VALLS, J.; REGUEIRO, S.; MORILLA, I.; URIBE, J. y OCHOA, C. (2017): "Cuidados paliativos en personas sin hogar", Anuario de psicología, noㅡ 47, pp. 95-106.

CASTEL, R. (1995): Les metamorphoses de la question sociale, Paris, Gallimard.

CORBETTA, P. (2010): Metodología y técnicas de investigación social, Madrid, MacGraw-Hill.

ESCOLAR, A. (2013): "Las desigualdades sociales y la salud en España”, Revista Temas, 218-219, pp. 64-66.

FEANTSA (2015): End-of-life cares for homeless people, Brussels, Feantsa [disponible en: 〈https://www.feantsa.org/en/feantsaposition/2015/07/03/feantsa-position-end-oflife-care-for-homeless-people?bcParent=27〉].

- (2017): ETHOS - European Typology on Homelessness and housing exclusion, Bruselas, Feantsa [disponible en <https://www.feantsa. org/en/toolkit/2005/04/01/ethostypology-on-homelessness-and-housingexclusion?bcParent $=27$ )].

FERNANDEZ, T. y PONCE DE LEON, L. (2018): Trabajo social individualizado. Metodología de intervención, Madrid, Ediciones Académicas.

FUNDACIÓN ARRELS (2017): ¿Puede ser que vivir en la calle afecte a la capacidad neurológica de la persona? [disponible en: 〈https://www. arrelsfundacio.org/es/puede-ser-que-vivir-enla-calle-afecte-a-la-capacidad-neurologica-dela-persona/>].

GARCÍA FERRANDO, M.; IBÁÑEZ, J. y ALVIRA, F. (1993): El análisis de la realidad social. Métodos y técnicas de investigación social. Madrid, Alianza.

GARCÍA, S. y ARÉVALO, D. (2015): “La intervención con población drogodependiente en situación de calle", Documentos de Trabajo Social, nํ56, pp. 94-112. 
INSTITUTO NACIONAL DE ESTADÍSTICA (2012): Encuesta a las personas sin hogar. [disponible en: 〈https:// www.ine.es/dyngs/INEbase/es/operacion.htm $? c=$ Estadistica_C\&cid $=1254736176817 \&$ menu $=$ resultados\&idp $=1254735976608$ >].

LLANO, J.C. (2019): La desigualdad en la salud, Madrid, European Anti-Poverty Network (EAPN-España), [disponible en: 〈https:// www.eapn.es/ARCHIVO/documentos/ documentos/1568024937_informe-pobreza-ysalud-vf.pdf>].

MARINA, P.A. (2018): “La baja exigencia: una manera de entender y cuidar a personas largo tiempo sin hogar", Cuadernos de psiquiatría comunitaria, vol. 15, n- 1, pp. 9-26.

MARLATT, G.A. (1996): "Harm reduction: Come as you are”, Addictive Behaviors, 21(6), 779-788.

MATULIČ, M.V.; DE VICENTE, I. y CAÏS, J. (2018): "Relatos de vida de personas sin hogar en la ciudad de Barcelona: desafiliación y salud", Comunitania, 16, pp. 141-157.

MINISTERIO DE SANIDAD Y POLÍTICA SOCIAL (2010): Análisis de situación para la elaboración de una propuesta de políticas e intervenciones para reducir las desigualdades sociales en salud en España, Madrid, Gobierno de España, [disponible en: 〈https://www.mscbs.gob.es/ profesionales/saludPublica/prevPromocion/ promocion/desigualdadSalud/docs/Analisis reducir_desigualdes.pdf`].

MINISTERIO DE SANIDAD, CONSUMO Y BIENESTAR SOCIAL (2015): Estrategia Nacional Integral para Personas sin Hogar 2015-2020, Madrid, Gobierno de España, [disponible en: 〈https:// www.mscbs.gob.es/ssi/familiasInfancia/ inclusionSocial/docs/ENIPSH.pdf»].

MORENO, G. (2009): “Características y tipologías de las personas sin hogar en Bizkaia. Hacia un enfoque complejo de la realidad del sinhogarismo", Zerbitzuan, 46, pp. 35-44.

MONCADA, S. (1997): “Factores de riesgo y protección en el consumo de drogas", en Plan Nacional sobre Drogas. Prevención de las drogodependencias, Madrid, Delegación del Gobierno para el Plan Nacional sobre Drogas, [disponible en: 〈http:// www.pnsd.mscbs.gob.es/profesionales/ publicaciones/catalogo/catalogoPNSD/ publicaciones/pdf/preven.pdf〉].

MUÑOZ, M; VÁZQUEZ, C. y VÁZQUEZ, J.J. (2003): Los límites de la exclusión. Estudio sobre los factores económicos, psicosociales y de salud que afectan a las personas sin hogar en Madrid, Madrid, Témpora.

ORGANIZACIÓN MUNDIAL DE LA SALUD (1946): Constitución de la Organización Mundial de la Salud, en Documentos Básicos. 48 Edición de 2014, Ginebra, Organización Mundial de la Salud, [disponible en: <https://apps.who.int/gb/bd/ PDF/bd48/basic-documents-48th-edition-sp. pdfs].

PANERO, S. y MUÑOZ, M. (2014): "Salud, calidad de vida y consumo de sustancias en función del tiempo en situación sin hogar", Anales de psicología, vol. 30, $\mathrm{n}^{0} 1$ (enero), pp. 70-77.

PÉREZ, J. y ABELLÁN, A. (2018): “Envejecimiento demográfico y vejez en España”, Panorama Social, 28, pp. 11-47.

ROCA, P.; PANADERO, S.; RODRÍGUEZ-MORENO, S.; MARTÍN, R.M. y VÁZQUEZ, J.J. (2019): “'Puerta giratoria' a la situación de sin hogar. Influencia de la salud, consumo de alcohol y padecimiento de sucesos vitales estresantes en el número de episodios en la situación sin hogar", Anales de psicología, vol. 35, 2 (mayo), pp. 175-180.

SÁNCHEZ MORALES, M. (1999): La población sin hogar en España: un caso extremo de exclusión social, Madrid, Sistema.

SEGURA, J. (2014): "Las desigualdades en salud: un reto para las políticas públicas”, Zerbitzuan, 55, pp. 47-63.

SUBIRATS, J. (Dir.) (2004): Pobreza y exclusión social, Barcelona, La Caixa.

- (2005): Análisis de los factores de exclusión social, Bilbao: BBVA.

TEZANOS, J.F. (2008): La sociedad dividida. Estructura de Clases y Desigualdades en las Sociedades Tecnológicas, Madrid, Biblioteca Nueva.

TORNERO, S.; FERNÁNDEZ, A. y CHARRIS, L. (2016): "Características de los ingresos hospitalarios de las personas sin hogar en Sevilla", Revista Española de Salud Pública, vol. 90, pp. 1-11.

URIBE, J. y ALONSO, S. (2009): Personas en situación de sin hogar en Barcelona: perfiles, estado de salud y atención sanitaria, Barcelona, Fundació Jaime Bofill.

VALLES, M.S. (2009): Técnicas cualitativas de investigación social, Madrid, Síntesis. 